\title{
Synthesis of Pincer-Type Bis(benzimidazolin-2-ylidene) Palladium Complexes and Their Application in C-C Coupling Reactions
}

\author{
F. Ekkehardt Hahn, ${ }^{*}$ Mareike C. Jahnke, and Tania Pape \\ Institut für Anorganische und Analytische Chemie der Westfälischen Wilhelms-Universität \\ Münster, Corrensstraße 36, D-48149 Münster, Germany
}

Supporting Information 
Molecular Structure of $\mathbf{2} \cdot \mathbf{M e O H}$. Crystals of $\mathbf{2} \cdot \mathrm{MeOH}$ suitable for an X-ray diffraction study have been obtained by slow evaporation of the solvent of a methanol solution of 2 . The dibenzimidazolium cation resides on a twofold axis which passes through $\mathrm{C} 12, \mathrm{C} 10$ and $\mathrm{Br}$ (Figure 1). The N1-C1 and N2-C1 bond distances [1.328(5) and 1.319(5) $\AA$ ] are equally long and fall in the range observed for similar benzimidazolium salts.

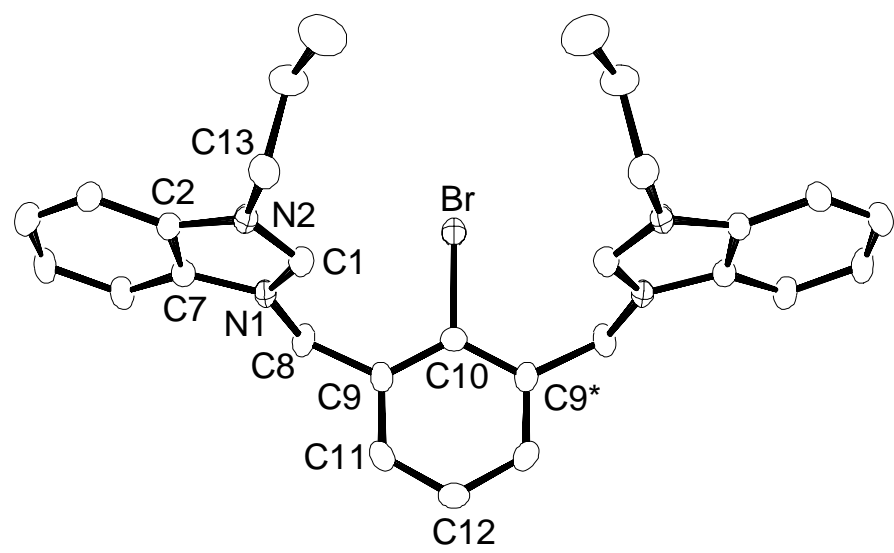

Figure S1. Molecular structure of the dibenzimidazolium cation of $\mathbf{2} \cdot \mathrm{MeOH}$. Hydrogen atoms have been omitted. Selected bond lengths $(\AA)$ and angles (deg): N1-C1 1.328(5), N1-C2 1.401(5), N1-C8 1.467(5), N2-C1 1.319(5), N2-C3 1.390(5), N2-C13 1.469(5), Br-C10 $1.916(6) ; \mathrm{N} 1-\mathrm{C} 1-\mathrm{N} 2$ 110.6(4).

General Method for the Heck Reactions. One of the palladium complexes 4-6 was dissolved in dimethylacetamide $(3 \mathrm{~mL})$. To this solution were added the aryl bromide (1.0 mmol), styrene or $n$-butyl acrylate $(1.4 \mathrm{mmol})$ and sodium acetate as base $(2.0 \mathrm{mmol})$. The solution was heated to $110^{\circ} \mathrm{C}$ while stirring. It was then allowed to cool to ambient temperature. Residual $\mathrm{NaOAc}$ and palladium complexes were removed by column chromatography with silica gel. The solution was then analyzed by quantitative GC chromatography (GC-FID) with a Shimadzu GC-2100 equipped with an Aglient Technologies HP 5 capillary column (30.0 m). 
Table S1. Heck Activity of 4-6 with Functionalized Aryl Bromides and Styrene ${ }^{a}$

\begin{tabular}{|c|c|c|c|c|c|}
\hline entry & catalyst & mol\% cat. & $\mathrm{R}$ & time, $\mathrm{h}$ & yield, \% \\
\hline 1 & 4 & 1.0 & $\mathrm{H}$ & 24 & 31.3 \\
\hline 2 & 5 & 1.0 & $\mathrm{H}$ & 24 & 40.7 \\
\hline 3 & 6 & 1.0 & $\mathrm{H}$ & 24 & 38.7 \\
\hline 4 & 4 & 1.0 & $\mathrm{C}(\mathrm{O}) \mathrm{H}$ & 24 & 100.0 \\
\hline 5 & 5 & 1.0 & $\mathrm{C}(\mathrm{O}) \mathrm{H}$ & 24 & 100.0 \\
\hline 6 & 6 & 1.0 & $\mathrm{C}(\mathrm{O}) \mathrm{H}$ & 24 & 100.0 \\
\hline 7 & 6 & 1.0 & $\mathrm{C}(\mathrm{O}) \mathrm{H}$ & 2 & 84.1 \\
\hline 8 & 6 & 0.1 & $\mathrm{C}(\mathrm{O}) \mathrm{H}$ & 2 & 55.1 \\
\hline 9 & 5 & 1.0 & $\mathrm{OCH}_{3}$ & 24 & 46.1 \\
\hline 10 & 6 & 1.0 & $\mathrm{OCH}_{3}$ & 24 & 50.4 \\
\hline 11 & 5 & 1.0 & $\mathrm{C}(\mathrm{O}) \mathrm{CH}_{3}$ & 24 & 98.5 \\
\hline 12 & 5 & 1.0 & $\mathrm{C}(\mathrm{O}) \mathrm{CH}_{3}$ & 2 & 61.3 \\
\hline 13 & 6 & 1.0 & $\mathrm{C}(\mathrm{O}) \mathrm{CH}_{3}$ & 24 & 97.0 \\
\hline 14 & 6 & 1.0 & $\mathrm{C}(\mathrm{O}) \mathrm{CH}_{3}$ & 2 & 60.0 \\
\hline
\end{tabular}

${ }^{a}$ Reaction conditions: $1.0 \mathrm{mmol}$ of the aryl bromide, $1.4 \mathrm{mmol}$ of styrene, 2.0 $\mathrm{mmol}$ of NaOAc, Pd catalyst and $3 \mathrm{~mL}$ of DMA. Yields were determined by gas chromatography.

General Method for the Suzuki Coupling. A two-necked 100mL flask was fitted with a reflux condenser and charged with an aryl bromide $(1 \mathrm{mmol})$, phenylboronic acid $(0.144 \mathrm{~g}, 1.2 \mathrm{mmol})$ and potassium carbonate $(0.276 \mathrm{~g}, 2 \mathrm{mmol})$. To this was added toluene $(50 \mathrm{~mL})$ and the suspension was brought to reflux. An aliquot of $1 \mathrm{~mL}$ of the dichloromethane solution of one of the palladium complexes 4-6 was injected to the boiling solution and the reaction mixture was heated under refluxed for the desired time. The mixture was then allowed to cool down to ambient temperature and the organic phase was subjected to column chromatography over a short silica gel column to remove the catalyst and remaining salts. The organic solution was then analyzed by quantitative GC analysis with a Shimadzu GC-2100 with an Aglient Technologies HP 5 capillary column $(30.0 \mathrm{~m})$. 
Table S2. Suzuki Activity of Complexes 4-6 with Functionalized Aryl Bromides ${ }^{a}$

$\begin{array}{llllll} & & & & \\ \text { entry } & \text { catalyst } & \text { mol\% cat. } & \mathrm{R} & \text { time, } \mathrm{h} & \text { yield, \% } \\ 1 & \mathbf{5} & 0.1 & \mathrm{H} & 24 & 75.8 \\ 2 & \mathbf{6} & 0.1 & \mathrm{H} & 24 & 79.9 \\ 3 & \mathbf{5} & 0.01 & \mathrm{H} & 24 & 64.9 \\ 4 & \mathbf{6} & 0.01 & \mathrm{H} & 24 & 67.7 \\ 5 & \mathbf{6} & 0.1 & \mathrm{C}(\mathrm{O}) \mathrm{H} & 24 & 100.0 \\ 6 & \mathbf{6} & 0.1 & \mathrm{C}(\mathrm{O}) \mathrm{H} & 2 & 50.7 \\ 7 & \mathbf{6} & 0.1 & \mathrm{C}(\mathrm{O}) \mathrm{K}_{2} \mathrm{CO}_{3} & 24 & 100.0 \\ 8 & \mathbf{6} & 0.1 & \mathrm{C}(\mathrm{O}) \mathrm{CH}_{3} & 2 & 48.7\end{array}$

${ }^{a}$ Reaction condition: $1.0 \mathrm{mmol}$ of aryl bromide, $1.2 \mathrm{mmol}$ of phenylboronic acid, $2.0 \mathrm{mmol}$ of $\mathrm{K}_{2} \mathrm{CO}_{3}$, Pd catalyst and $50 \mathrm{~mL}$ of toluene. Yields were determined by gas chromatography.

General Procedure for the Preparation of 2,6-Bis-( $N$-alkyl- $N^{\prime}$-methylenebenzimidazolium)-1-bromophenylene Dibromides (1-3). A solution of 2,6-bis(bromomethyl)1-bromophenylene $(1.0 \mathrm{mmol})$ and an $\mathrm{N}$-alkylated benzimidazole derivative $(2.2 \mathrm{mmol})$ in 1,4 dioxane $(50 \mathrm{~mL})$ was heated under reflux for $16 \mathrm{~h}$. The solvent was removed and the solid residue was washed twice with THF (5 mL each). Normally white solid were obtained which were dried in vacuo. Correct elemental analyses for the hygroscopic benzimidaziolium salts were difficult to obtain.

\section{2,6-Bis-( $N$-ethyl- $N^{\prime}$-methylenebenzimidazolium)-1-bromophenylene $\quad$ Dibromide}

Yield: $86.2 \% .{ }^{1} \mathrm{H}$ NMR (300.1 MHz, DMSO- $\left.d_{6}\right): \delta 10.08(\mathrm{~s}, 2 \mathrm{H}, \mathrm{NCHN}), 8.20-8.13(\mathrm{~m}, 2 \mathrm{H}$, Ar-H), 7.96 - 7.90 (m, 2H, Ar-H), 7.76 - 7.62 (m, 4H, Ar-H), 7.46 (s, br, 3H, Ph-H), 5.94 (s, 4H, $\left.\mathrm{NCH}_{2} \mathrm{Ph}\right), 4.62\left(\mathrm{q},{ }^{3} \mathrm{~J}=7.2 \mathrm{~Hz}, 4 \mathrm{H}, \mathrm{NCH}_{2} \mathrm{CH}_{3}\right), 1.56\left(\mathrm{t},{ }^{3} \mathrm{~J}=7.2 \mathrm{~Hz}, 6 \mathrm{H}, \mathrm{NCH}_{2} \mathrm{CH}_{3}\right) .{ }^{13} \mathrm{C} \mathrm{NMR}$ (75.1 MHz, DMSO- $\left.d_{6}\right): \delta 143.2(\mathrm{NCHN}), 134.5,131.5(\mathrm{Ph}-\mathrm{C}), 131.4,131.3(\mathrm{Ar}-\mathrm{C}), 129.1(\mathrm{Ph}-$ C), 127.2, 127.1 (Ar-C), 124.9 (Ph-C), 114.4, 114.2 (Ar-C), $51.1\left(\mathrm{NCH}_{2} \mathrm{Ph}\right), 42.7\left(\mathrm{NCH}_{2} \mathrm{CH}_{3}\right)$, $14.7\left(\mathrm{NCH}_{2} \mathrm{CH}_{3}\right)$. MS (MALDI): $\left.m / z 554\left([\mathrm{M}-\mathrm{Br}]^{+}\right), 475[\mathrm{M}-2 \mathrm{Br}]^{+}\right)$. Anal. Calcd for $\mathrm{C}_{26} \mathrm{H}_{27} \mathrm{~N}_{4} \mathrm{Br}_{3}$ (635.2): C, 49.16; H, 4.28; N, 8.82. Found: C, 48.66; H, 4.17; N, 8.58. 
Yield: $89.6 \%$. ${ }^{1} \mathrm{H}$ NMR (300.1 MHz, DMSO- $\left.d_{6}\right): \delta 10.06(\mathrm{~s}, 2 \mathrm{H}, \mathrm{NCHN}), 8.21-8.15(\mathrm{~m}, 2 \mathrm{H}$, Ar-H), $7.97-7.91$ (m, 2H, Ar-H), 7.75 - 7.63 (m, 4H, Ar-H), 7.51 (s, br, 3H, Ph-H), 5.94 (s, 4H, $\left.\mathrm{NCH}_{2} \mathrm{Ph}\right), 4.56\left(\mathrm{t},{ }^{3} \mathrm{~J}=7.2 \mathrm{~Hz}, 4 \mathrm{H}, \mathrm{NCH}_{2} \mathrm{CH}_{2} \mathrm{CH}_{3}\right), 1.94$ (sext, ${ }^{3} \mathrm{~J}=7.2 \mathrm{~Hz}, 4 \mathrm{H}, \mathrm{NCH}_{2} \mathrm{CH}_{2} \mathrm{CH}_{3}$ ), $0.91\left(\mathrm{t},{ }^{3} \mathrm{~J}=7.2 \mathrm{~Hz}, 6 \mathrm{H}, \mathrm{NCH}_{2} \mathrm{CH}_{2} \mathrm{CH}_{3}\right) .{ }^{13} \mathrm{C} \mathrm{NMR}\left(75.1 \mathrm{MHz}, \mathrm{DMSO}-d_{6}\right): \delta 143.4(\mathrm{NCHN})$, 134.5), 131.7 (Ph-C), 131.6, 131.5 (Ar-C), 129.2 (Ph-C), 127.3, 127.2 (Ar-C), 125.2 (Ph-C), 114.4, 114.2 (Ar-C), $51.2\left(\mathrm{NCH}_{2} \mathrm{Ph}\right), 48.6\left(\mathrm{NCH}_{2} \mathrm{CH}_{2} \mathrm{CH}_{3}\right), 22.5\left(\mathrm{NCH}_{2} \mathrm{CH}_{2} \mathrm{CH}_{3}\right), \quad 11.0$ $\left(\mathrm{NCH}_{2} \mathrm{CH}_{2} \mathrm{CH}_{3}\right)$. MS (MALDI): $\left.m / z 582\left([\mathrm{M}-\mathrm{Br}]^{+}\right), 503[\mathrm{M}-2 \mathrm{Br}]^{+}\right)$. Anal. Calcd for $\mathrm{C}_{28} \mathrm{H}_{31} \mathrm{~N}_{4} \mathrm{Br}_{3}$ (663.3): C, 50.70; H, 4.71; N, 8.45. Found: C, 50.45; H, 4.48; N, 8.49.

2,6-Bis-( $N$-butyl- $N^{\prime}$-methylenebenzimidazolium)-1-bromophenylene $\quad$ Dibromide (3). Yield: $91.2 \% .{ }^{1} \mathrm{H}$ NMR (300.1 MHz, DMSO- $\left.d_{6}\right): \delta 10.10(\mathrm{~s}, 2 \mathrm{H}, \mathrm{NCHN}), 8.21-8.13$ (m, 2H, Ar-H), 7.99 - 7.90 (m, 2H, Ar-H), 7.73 - 7.62 (m, 4H, Ar-H), 7.51 (s, br, 3H, Ph-H), 5.94 (s, 4H, $\mathrm{NCH}_{2} \mathrm{Ph}$ ), $4.60\left(\mathrm{t},{ }^{3} J=7.2 \mathrm{~Hz}, 4 \mathrm{H}, \mathrm{NCH}_{2} \mathrm{CH}_{2} \mathrm{CH}_{2} \mathrm{CH}_{3}\right.$ ), 1.91 (quint, ${ }^{3} J=7.2 \mathrm{~Hz}, 4 \mathrm{H}$, $\mathrm{NCH}_{2} \mathrm{CH}_{2} \mathrm{CH}_{2} \mathrm{CH}_{3}$ ), 1.35 (sext, $\left.{ }^{3} J=7.2 \mathrm{~Hz}, 4 \mathrm{H}, \mathrm{NCH}_{2} \mathrm{CH}_{2} \mathrm{CH}_{2} \mathrm{CH}_{3}\right), 0.91\left(\mathrm{t},{ }^{3} J=7.2 \mathrm{~Hz}, 6 \mathrm{H}\right.$, $\left.\mathrm{NCH}_{2} \mathrm{CH}_{2} \mathrm{CH}_{2} \mathrm{CH}_{3}\right) .{ }^{13} \mathrm{C}$ NMR (DMSO-d 6 ): $\delta 143.4$ (NCHN), 134.5, $131.7(\mathrm{Ph}-\mathrm{C}), 131.6,131.5$ (Ar-C), 129.2 (Ph-C), 127.3, 127.2 (Ar-C), 125.2 (Ph-C), 114.4, 114.2 (Ar-C), $51.2\left(\mathrm{NCH}_{2} \mathrm{Ph}\right)$, $47.0 \quad\left(\mathrm{NCH}_{2} \mathrm{CH}_{2} \mathrm{CH}_{2} \mathrm{CH}_{3}\right), \quad 31.0 \quad\left(\mathrm{NCH}_{2} \mathrm{CH}_{2} \mathrm{CH}_{2} \mathrm{CH}_{3}\right), \quad 19.4 \quad\left(\mathrm{NCH}_{2} \mathrm{CH}_{2} \mathrm{CH}_{2} \mathrm{CH}_{3}\right), \quad 13.8$ $\left(\mathrm{NCH}_{2} \mathrm{CH}_{2} \mathrm{CH}_{2} \mathrm{CH}_{3}\right)$. MS (MALDI): $m / z 610\left([\mathrm{M}-\mathrm{Br}]^{+}\right), 529\left([\mathrm{M}-2 \mathrm{Br}]^{+}\right)$. Anal. Calcd for $\mathrm{C}_{30} \mathrm{H}_{35} \mathrm{~N}_{4} \mathrm{Br}_{3}$ (691.4): C, 52.12; H, 5.10; N, 8.10. Found: C, 51.66; H, 4.82; N, 7.92.

X-ray Crystallography. Diffraction data for $\mathbf{2} \cdot \mathrm{MeOH}$ were collected on a Bruker AXS APEX CCD diffractometer equipped with a rotation anode at 123(2) K using graphite-monochromatic MoK $\alpha$ radiation $(\lambda=0.71073 \AA)$. For compound $\mathbf{6}$ the data were obtained on a Bruker AXS APEX system with a rotating anode using $\mathrm{CuK} \alpha$ radiation $(\lambda=1.54184 \AA$ ) at $120(2) \mathrm{K}$. Data were collected over the full sphere and were corrected for absorption. The data reduction was performed with the Bruker SMART ${ }^{1}$ program package. For further crystal and data collection details see Table 3. All crystal structures were solved by using SHELXS-97 ${ }^{2}$ by heavy-atom 
methods and refined with SHELXL-97 $7^{3}$ against $F^{2}$ using first isotropic and later anisotropic thermal parameters for all none hydrogen atoms. Hydrogen positions were calculated and thermally fixed to 1.3 Ueqv of the parent atom.

Table S3. Summary of Crystallographic Data for $2 \cdot \mathrm{MeOH}$ and 6

\begin{tabular}{|c|c|c|}
\hline & $\mathbf{2} \cdot \mathrm{MeOH}$ & 6 \\
\hline formula & $\mathrm{C}_{29} \mathrm{H}_{35} \mathrm{~N}_{4} \mathrm{Br}_{3} \mathrm{O}$ & $\mathrm{C}_{30} \mathrm{H}_{33} \mathrm{~N}_{4} \mathrm{BrPd}$ \\
\hline$M_{\mathrm{r}}$ & 695.34 & 635.91 \\
\hline cryst size $[\mathrm{mm}]$ & $0.13 \times 0.13 \times 0.04$ & $0.27 \times 0.12 \times 0.03$ \\
\hline$a[\AA]$ & $19.590(3)$ & $8.68300(10)$ \\
\hline$b[\AA]$ & $20.710(3)$ & $10.7218(2)$ \\
\hline$c[\AA]$ & $7.1575(10)$ & $15.8253(3)$ \\
\hline$\alpha[\mathrm{deg}]$ & 90 & $102.4340(10)$ \\
\hline$\beta[\operatorname{deg}]$ & $94.768(3)$ & $94.2730(10)$ \\
\hline$\gamma[\mathrm{deg}]$ & 90 & $113.3970(10)$ \\
\hline$V\left[\AA^{3}\right]$ & 2893.8(7) & $1299.22(4)$ \\
\hline$Z$ & 4 & 2 \\
\hline space group & $C 2 / m$ & $P \overline{1}$ \\
\hline$\rho_{\text {calcd }}\left[\mathrm{g} \mathrm{cm}^{-3}\right]$ & 1.596 & 1.626 \\
\hline$\mu\left[\mathrm{mm}^{-1}\right]$ & $4.211(\operatorname{MoK} \alpha)$ & $7.760(\mathrm{CuK} \alpha)$ \\
\hline $2 \theta$ range $[\mathrm{deg}]$ & $2.8-50.00$ & $11.2-142.1$ \\
\hline data collected & 11798 & 7478 \\
\hline unique data & 2637 & 4308 \\
\hline obsd data $[I \geq 2 \sigma(I)]$ & 2195 & 3672 \\
\hline$R$ (all data) & 0.0519 & 0.0512 \\
\hline wR (all data) & 0.1010 & 0.1221 \\
\hline no. of variables & 182 & 327 \\
\hline peak/hole [e $\AA^{3}$ ] & $1.557,-0.939$ & $1.417,-0.857$ \\
\hline
\end{tabular}

\section{References}
(1) SMART, Bruker AXS 2000.
(2) SHELXS-97, Sheldrick, G. M. Acta Cryst. 1990, A46, 467.
(3) Sheldrick, G. M. SHELXL-97, Universität Göttingen: Germany, 1997. 